\title{
Política pública de fomento al emprendimiento juvenil para el desarrollo local en el municipio de Colima 2009-2013
}

\author{
Rosario Cota Yañez ${ }^{1}$ \\ https://orcid.org/0000-0002-1866-7191 \\ Mayra Rivera Escareño ${ }^{2}$
}

\section{RESUMEN}

Los cambios estructurales en los últimos años han traído transformaciones en la dinámica social y económica del desarrollo local. Uno es el desempleo, el objetivo del presente documento consiste en analizar la manera en que las políticas públicas para el emprendimiento juvenil contribuyen para el desarrollo local. Dentro de los principales autores citados sobre la temática destaca: González (2007), Vázquez (2008), Gallicchio (2004), Aguilar, Mariñez y Garza (2009), Balaguer, Hidalgo, Pérez y Pérez (2009) y Valencia (2012) entre otros. La metodología consistió en aplicar una encuesta a 21 beneficiarios del programa de apoyo a jóvenes emprendedores en el municipio de Colima. Los principales resultados encontrados fue que en su mayoría son solteros, con alta escolaridad (nivel licenciatura) en el área de administración y finanzas, sus edades oscilan entre los 23 y 26 años; establecen redes de cooperación en dos niveles: a nivel familiar (familia nuclear) y personal (amigos y conocidos). Se enfocaron al sector comercio y servicios; tienen una tradición familiar de emprendimiento y en promedio crearon 3 empleos por empresa.

Palabras clave: Política pública, Emprendimiento, Jóvenes, Colima.

Política pública de promoção do empreendedorismo juvenil para o desenvolvimento local no município de Colima 2009-2013

\section{RESUMO}

As mudanças estruturais nos últimos anos, trouxeram transformações na dinâmica social e econômica do desenvolvimento local. Um deles é o desemprego, o objetivo deste documento é analisar como as políticas públicas para o empreendedorismo juvenil contribuem para o desenvolvimento local. Entre os principais autores citados sobre o assunto, destacam-se: González (2007), Vázquez (2008), Gallicchio (2004), Aguilar, Mariñez e Garza (2009), Balaguer, Hidalgo, Pérez e Pérez (2009), Valencia (2012), entre outros. A metodologia consistiu na aplicação de um levantamento de 21 beneficiários do programa para apoiar jovens empreendedores no município de Colima. Os resultados encontrados indicam que em sua maioria, são solteiros, com ensino médio (nível de licença) na área de administração e finanças, suas idades variam de 23 a 26 anos; estabelecer redes de cooperação em dois níveis: a nível familiar (família nuclear) e pessoal (amigos e conhecidos). Centraram-se no sector do comércio e dos serviços; tem uma tradição familiar de empreendedorismo e, em média, criaram 3 empregos por empresa.

Palavras-chave: Políticas Públicas, Empreendedorismo, Juventude, Colima.

Public policy to promote youth entrepreneurship for local development in the municipality of Colima 2009-2013

\section{ABSTRACT}

Structural changes in recent years have brought transformations in the social and economic dynamics of local development. First is unemployment, the objective of this document is to analyze the way in which public policies for young entrepreneurship contribute to local development. Among the main authors cited on the subject highlights: González (2007); Vázquez (2008); Gallicchio (2004); Aguilar, Mariñez and Garza (2009); Balaguer, Hidalgo, Pérez and Pérez (2009) and Valencia (2012); among others. The methodology consisted of applying a survey to 21 beneficiaries of the support program for young entrepreneurs in the municipality of Colima. The main results found were that they are mostly single, with high schooling (undergraduate level) in the area of administration and finance, their ages range between 23 and 26 years; establish cooperation networks at two levels: at family level (nuclear family) and personal (friends and acquaintances). They focused on the commerce and services sector; They have a family tradition of entrepreneurship and on average they created 3 jobs per company.

\footnotetext{
${ }^{1}$ Universidad de Guadalajara (UdG), México. Correo electronico: macotaya@gmail.com

${ }^{2}$ Universidad de Guadalajara (UdG), México.
} 
Keywords: Public policy, Entrepreneurship, Youth, Colima.

\section{Introducción}

Uno de los principales problemas a los que se enfrentan los jóvenes actualmente al concluir sus estudios, es la restricción de alternativas para emplearse (González, 2007; Mora y De Oliveira 2011). El mercado de trabajo cada día es más selectivo. Se tienen que buscar nuevas alternativas de empleo. El presente documento analiza la manera en que la implementación de políticas públicas para el fomento del emprendimiento juvenil ha contribuido al desarrollo de la localidad. ¿En qué consisten? Y ¿cuáles son las formas de beneficiarse? Para ello, primeramente se recurrió a la Secretaria de la Juventud (SEJUV) de Colima con el fin de solicitar el padrón de beneficiados, que fue proporcionado de manera incompleta, ya que la Ley de Protección de Datos no permite dar los domicilios de los negocios creados. Fue necesario establecer un rastreo por cuenta propia de los 54 registrados. De los que se ubicaron 30. La localización de los negocios se llevó a cabo mediante las redes sociales principalmente Facebook que es donde los jóvenes promocionan sus productos y servicios. Otra forma de localización fue a través de directorios electrónicos de servicios, al estar actualizados dieron certeza de la ubicación de los negocios. Se visitaron los 30 establecimientos y se encontró que cinco ya no estaban en funciones, tres estaban a cargo de empleados y no accedieron a proporcionar datos del titular. Y en dos más no quisieron cooperar. Quedando al final un total de 21.

Para recabar la información se llevó a cabo un cuestionario que consta de 20 preguntas enfocadas a rescatar la experiencia del joven emprendedor, tanto con el programa de la SEJUV, como en el plano personal.

La hipótesis toma en consideración que las políticas públicas de fomento al emprendimiento juvenil son un factor de desarrollo local, ya que fomentan una mayor participación de los jóvenes y con ello mayor creación de empresas lo cual contribuye al incremento de las fuentes de empleo e ingresos, que a la larga pudieran propiciar mejores condiciones de vida y contribuir al desarrollo de las localidades.

El documento se encuentra estructurado de cuatro partes: la primera expone las consideraciones en torno a las transformaciones productivas, posteriormente se habla sobre la situación de los jóvenes en México, en seguida se presentan las características de los jóvenes emprendedores y por último las consideraciones finales.

\section{Contexto de las transformaciones productivas}

Desde los años 70's, los gobiernos y la sociedad han experimentado cambios estructurales, resultando nuevas dinámicas y relaciones. La implementación del libre mercado así como las políticas y nuevas formas de contratación de las empresas (outsourcing, pago por comisión) por lo que el trabajador va perdiendo paulatinamente sus derechos laborales adquiridos como el empleo fijo, pensión por años de antigüedad, prestaciones sociales, entre otros. Las nuevas generaciones se ven obligadas a estructurar otras formas de emplearse mediante la creación de emprendimientos locales.

Los jóvenes son quienes mayormente se han visto perjudicados (cuyas edades están entre los 18 y 29 años). Las oportunidades de insertarse en un empleo digno son cada vez más difíciles, independientemente de su preparación académica. A la par de estos acontecimientos, se empieza a reconocer y aplicar la intervención de conceptos "de abajo hacia arriba", de lo local a lo nacional. Esta tendencia resalta las cualidades y los recursos de los territorios, trata de incidir de manera positiva en su entorno mediante un impulso al desarrollo local (García-García, 2015; Várguez, 2010).

Entendiéndose al desarrollo local como un proceso de crecimiento económico y cambio estructural en constante cambio, que utiliza las potencialidades propias del territorio, los actores locales deciden las acciones que les son favorables para incrementar su propio bienestar y de la comunidad (Vázquez, 2008). La generación de empleo es uno de los principales ejes del desarrollo, 
a través de esta actividad remunerada se logran cubrir las necesidades de sobrevivencia, dignificación y al mismo tiempo se está contribuyendo a la actividad económica del territorio.

El empleo por su parte, debe constituir un eje en las estrategias de desarrollo (...). El mismo no es solamente dependiente del crecimiento económico, sino que deben existir políticas y acciones que lo vinculen fuertemente a la calidad de vida y a la apropiación por parte de los ciudadanos y los territorios (Gallicchio, 2004:21).

El Estado como actor ha buscado soluciones a la problemática respondiendo con la implementación de políticas públicas de fomento al emprendimiento. Cuya finalidad es desarrollar actividades económicas con miras a la creación de empresas y negocios, que aprovechan las potencialidades y habilidades de las personas, asumen un riesgo, se espera que esta acción tenga incidencia en la sociedad, estimulando la creación de empleos y el crecimiento económico (Balaguer, Hidalgo, Pérez y Pérez, 2009).

Para que las acciones emprendidas tengan éxito es necesario la implementación de una política pública la cual se compone de acciones u omisiones por parte de la autoridad (Mora y De Oliveira, 2016; Cardozo, 1993). El éxito estará directamente relacionado con el grado de identificación y respuesta de la sociedad, entendidas como: un conjunto (secuencia, sistema, ciclo) de acciones, estructuradas en modo intencional y causal, que se orientan a realizar objetivos considerados de valor para todos o también resolver problemas cuya solución es considerada de interés o beneficio público (Aguilar, Mariñez y Garza, 2009).

La estrategia para combatir el desempleo juvenil ha respondido principalmente al autoempleo, a la generación e impulso de las micro y pequeñas empresas, para que los jóvenes no sólo logren cumplir sus objetivos personales, sino que a la par logren generar empleos para otros jóvenes mediante el emprendimiento (Valencia, 2012:105). El gobierno en los últimos años ha dado impulso al desarrollo de programas a las microempresas en un intento de generar mayor participación de los ciudadanos que contribuyan mediante iniciativas locales apoyadas con fondos gubernamentales.

\section{Situación de los jóvenes en México}

Hoy en día México se enfrenta a una crisis de empleo juvenil decente de altas proporciones, aun cuando en las naciones integrantes de la Organización para la Cooperación y el Desarrollo Económicos (OCDE), es la cuarta con los indicadores de desempleo más bajos, $4.9 \%$ de la Población Económicamente Activa (PEA) para el año 2014 se ubicaba en dicha condición, la tasa de desempleo juvenil para el mismo periodo de medición alcanzó el 9.6\%, es decir, duplicó la tasa de desempleo general (OCDE, 2014). Datos de la Encuesta Nacional de Ocupación y Empleo (ENOE), (2013) del INEGI indicaron que el 33\% de jóvenes de estratos altos con estudios profesionales terminados, laboraban en ocupaciones no profesionales, mientras los jóvenes de estratos bajos con estudios profesionales terminados, el 60\% laboraba en ocupaciones no profesionales.

México se ha mantenido, ya que aun cuando el porcentaje de desocupados han disminuido, quienes se ubican en la informalidad global han aumentado considerablemente respecto al año 2013: La tasa de desocupación de jóvenes durante el primer trimestre del año 2014 fue del 8.4\%. En lo que respecta a la informalidad global en el mismo periodo, se reporta que el $67 \%$ de la población joven está en dicha condición.

Por su parte Colima es uno de los Estados de la República mexicana que presenta las tasas más altas $(8.28 \%)$ de desocupación juvenil precedido por trece estados de un total de 32 como son: Durango, Hidalgo, Sinaloa, Chihuahua, Sonora, Aguascalientes, Tlaxcala, Coahuila, Guanajuato, Estado de México, Nuevo León, D.F y Tamaulipas (ENOE, 2013).

El sector poblacional presenta demandas y problemáticas particulares, de acuerdo al Plan Estatal de Desarrollo Colima 2009-2015, la falta de oportunidades de empleo entre la juventud, es una de las principales; para el año 2010 la población joven económicamente inactiva fue de 96 mil 256 
jóvenes, de los cuales el 20.4\% no trabajaba ni estudiaba, representando alrededor de 19 mil 637 jóvenes, el 10.4\% del total de la población joven (PEDC, 2015).

Los gobiernos, asociaciones civiles, universidades, han tratado de dar respuesta a esta problemática fomentando el emprendimiento a través del autoempleo, de la micro y pequeña empresa. El desempleo en el Estado de Colima ha crecido gradualmente necesita generar 13 mil empleos anuales para lograr la inclusión de los jóvenes que están listos para el mercado laboral (PEDC, 2015).

La situación en el municipio de Colima no es diferente, concentra el 22\% de la población total estatal entre los 15 y los 29 años. El municipio tiene un gran reto, puesto que sus jóvenes no encuentran los espacios suficientes para desarrollarse laboralmente. Según datos de la Encuesta Nacional de Ocupación y Empleo (ENOE, 2013) en el primer trimestre del año 2012, en el municipio de Colima se localizó poco más del $43 \%$ de los jóvenes desocupados del total de la entidad.

De acuerdo a los datos del municipio de Colima existe una incidencia de autoempleo y emprendimiento del $21 \%$ es decir, que de la población económicamente activa este porcentaje desarrolla una actividad laboral por cuenta propia la gran mayoría de la población aún se encuentra bajo el esquema del empleo.

Uno de los principales programas es "Emprendedores Juveniles", operado y ejecutado por la Secretaria de la Juventud Estatal (SEJUV). Este programa consiste en la creación o fortalecimiento de empresas ya existentes, va dirigido a jóvenes de entre 18 y 29 años. Cuyo principal apoyo es la ayuda técnica para la formulación del proyecto de negocio, posteriormente se hace un seguimiento donde el joven puede tener acceso a un crédito de $\$ 18,000$ o $\$ 36,000$ pesos, dependiendo el tipo de proyecto (SEJUV, 2013). La asesoría es brindada por la SEJUV mediante la incubadora de empresas, que recientemente fue reconocida por el Banco Interamericano de Desarrollo en el marco del Sexto Congreso Mundial de Oportunidades Económicas para Jóvenes.

El modelo de incubación permite a los jóvenes interesados, la formulación de un plan de negocios autosuficiente, que puede orientarse a las actividades tradicionales del territorio o incluso al desarrollo e implementación de tecnología intermedia y alta tecnología. Uno de los requisitos es la posibilidad de generar empleo, no solo para el joven sino para otros jóvenes del municipio, las empresas que se han creado generan 2 empleos en promedio. Los proyectos son evaluados por un comité técnico, enfocándose en tres aspectos: a) Viabilidad técnica, financiera y comercial del proyecto, b) Generación de empleos (de 1 a 3) y c) Demostrar rentabilidad. Una vez seleccionados los proyectos, los jóvenes reciben asesoría y acompañamiento de manera que la recién creada empresa se consolide y logre expandirse en empleos y ganancia (SEJUV, 2013).

Otro de los programas de fomento a la cultura emprendedora es "Jóvenes Emprendedores", consiste en un curso-taller desarrollado por una universidad privada, el Tecnológico de Monterrey y el Instituto Politécnico Nacional, por encargo de la Secretaría de Economía. Este programa también es operado a través de la SEJUV (2013).

El modelo tiene como principal herramienta el taller "Yo Emprendo", el cual costa de 150 horas, 72 son presenciales y 78 en línea; durante las primeras, el emprendedor tiene la oportunidad de recorrer los 10 módulos del taller con actividades, temas interesantes y dinámicas vivenciales. En las 78 horas en línea los emprendedores tienen la tarea de desarrollar su plan de negocios real o simulado en la plataforma de jóvenes emprendedores; todo lo anterior es llevado a cabo con el acompañamiento de un facilitador, el cual está autorizado y capacitado por la secretaría de economía (SEJUV, 2013).

El taller tiene como objetivo fomentar el espíritu emprendedor en los jóvenes a través del desarrollo de habilidades, actitudes y valores empresariales, para concretar planes de negocios que se constituyan en un semillero de empresas a través del acompañamiento especializado de facilitadores y asesores, que les brindan herramientas para la incorporación en el ambiente emprendedor-empresarial, logrando así contribuir al desarrollo económico y social del territorio. 
Durante el taller, a los participantes se les manifiesta la posibilidad de acreditar el mismo con un proyecto que les permita participar posteriormente dentro de la incubadora y en su caso poder ser beneficiados con asesoría y vinculación a fuentes de financiamiento.

"Yo Emprendo" desde su implementación ha tenido un notable incremento, en el año 2010 se registró la participación de 300 jóvenes, para el año 2011 fueron 400, en el 2012 se dio un incremento significativo con la participación de 1200 jóvenes (SEJUV, 2013).

En la tabla 1 se muestran los programas encaminados a la promoción del emprendimiento juvenil (Tabla 1).

\begin{tabular}{|c|l|}
\hline \multicolumn{2}{|c|}{ Tabla 1 Programas de fomento del emprendimiento juvenil, municipio de Colima 2013} \\
\hline PROGRAMA & \multicolumn{1}{c|}{ DESCRIPCIÓN } \\
\hline ¿Por qué emprender? & $\begin{array}{l}\text { Pláticas y conferencias impartidas por expertos en la materia, cuyos principales } \\
\text { beneficiados son jóvenes de entre 12 y } 29 \text { años. }\end{array}$ \\
\hline $\begin{array}{c}\text { Taller "Yo } \\
\text { emprendedor" }\end{array}$ & $\begin{array}{l}\text { Capacitación a jóvenes mayores entre los 18 y los 29 años cuyo objetivo es } \\
\text { formar emprendedores para desarrollar conocimientos, habilidades y actitudes } \\
\text { empresariales que les permitan tener proyectos listos para incubación. }\end{array}$ \\
\hline $\begin{array}{c}\text { Incubadora de } \\
\text { Empresas }\end{array}$ & $\begin{array}{l}\text { Enfocado a jóvenes entre 18 y 29 años, se ofrece asesoría y acompañamiento } \\
\text { especializado en el desarrollo de planes de negocios a emprendedores que ya } \\
\text { tienen un producto o servicio definido y quieren crear su propia empresa } \\
\text { aprovechando los beneficios de la aplicación del modelo de incubación } \\
\text { acreditado. }\end{array}$ \\
\hline $\begin{array}{c}\text { Financiamiento a } \\
\text { jóvenes } \\
\text { emprendedores }\end{array}$ & $\begin{array}{l}\text { Enfocado a jóvenes entre 18 y 29 años que cuentan con un plan de negocios, } \\
\text { los montos de financiamiento van desde 18 mil hasta 36 mil pesos. }\end{array}$ \\
\hline $\begin{array}{c}\text { Servicios de } \\
\text { consultoría a } \\
\text { empresarios }\end{array}$ & Talleres y asesorías de que dispone la incubadora de empresas. \\
\hline
\end{tabular}

Fuente: Elaboración propia con base solicitud de información SEJUV, 2013.

Características de los jóvenes emprendedores en Colima de acuerdo a la encuesta

La metodología consistió en la aplicación de una encuesta a 21 jóvenes beneficiarios del programa que representan el total del listado de la SEJUV. Se implementaron veinte reactivos cuyas variables fueron: género, edad, lugar de residencia, actividad que realizaban anteriormente, desempeño de otras actividades, tiempo de iniciar el negocio, motivos para empezar en un negocio propio, factores al momento de la elección, si cuentan con socios, la posesión del local, apoyos recibidos por parte de las instituciones gubernamentales, medio por lo que se enteró del programa, cómo considera el apoyo recibido, importancia del apoyo, si acudió a otras fuentes de apoyo, dificultades para el desarrollo del negocio, percepciones de la calidad de vida a partir de crear el negocio, si recibió apoyo familiar, número de personas empleadas, cuenta con sucursales, y planes de expansión a futuro en las misma actividad.

La aplicación del instrumento se llevó a cabo en los domicilios de los jóvenes, tuvieron una duración de 30 a 40 minutos.

Se encontró que tanto hombres como mujeres se sienten en las mismas condiciones de participación, pero también con la capacidad de llevar a cabo un proyecto de emprendimiento propio. Presentan una escolaridad alta el 62\% tienen licenciatura y 10\% posgrado. Enfocándose hacia las carreras de administración y finanzas. El rango de edad oscila entre los 23 y 26. Situación que refuerza los resultados obtenidos por García-García (2015: 232) ya que el 75\% de estudiantes universitarios encuestados de las carreras de administración y Gestión de Pymes mencionaron que en un lapso de dos años podrían empezar un negocio. En cuanto a la actividad que los jóvenes desarrollaban anterior a la apertura del negocio, destaca con un $67 \%$ estar estudiando, lo cual viene a refrendar lo anteriormente expuesto. 
El 33\% se encontraba laborando con calidad de empleado, en las actividades que posteriormente desarrollarían de manera propia. Es decir, la experiencia laboral les ayudó a adquirir el conocimiento sobre el rubro en el cual desarrollarían sus negocios. Incluso, en algunos casos fueron sus propios patrones quienes los motivaron a generar sus propios negocios, ya sea orientándolos hacia los apoyos disponibles, o inculcándoles la idea de incursionar en el emprendimiento.

Por lo tanto, no es de sorprender que el $52 \%$ de los jóvenes respondieran que eligieron el giro de sus respectivos negocios por los conocimientos previos que tenían de esa actividad, contra un 38\% que eligió por que la actividad le resultaba de su agrado. Cifra similar a la encontrada por GarcíaGarcía (2015:1232) en el 49\% de los casos mencionaron que lo más importante era contar con habilidades personales por ejemplo en la creación de Planes de Negocios, así como generar bienes o servicios con potencial para auto emplearse y generar un negocio propio.

Una característica importante que destaca entre los jóvenes emprendedores, es que, aunque se les planteó como opción de respuesta la elección del negocio a partir de la rentabilidad que pudiera representar, solo uno respondió que eligió la actividad por esta razón. Por ello las ganancias inmediatas no resultan ser un aliciente para los jóvenes.

Prefieren desarrollar actividades que les satisfagan en otros rubros más allá del económico, mencionan que al realizar bien lo que saben y les gusta, los resultados se verán reflejados con el tiempo. Porque al estar en un negocio que no les satisfaga, así estén recibiendo buenas ganancias, a la larga será tan tedioso como estar en un empleo. Argumento contrario a lo encontrado por García-García (2015:1232) ya que el motivo principal para iniciar un negocio propio fue el incremento de los ingresos.

La mayoría de los jóvenes mencionan que tenían planes de iniciar un negocio, pero que no lo vislumbraban al corto plazo, fueron diversas razones las que los motivaron a buscarlo de manera inmediata. Una de ellas fue la facilidad del programa de la SEJUV, aunado a la necesidad de empezar a generar sus ingresos, después de egresar de sus respetivos niveles de estudios el colocarse en el mercado laboral no era fácil.

También destacan las respuestas, de no querer ser empleado, por las malas condiciones que ello representa, pero también por la dificultad de colocarse en puestos que puedan satisfacer sus necesidades, así como planteárselo como un plan de vida, hacer del emprendimiento algo más que una fuente de ingresos.

Otro factor que comparten los jóvenes emprendedores es la influencia de su entorno, específicamente de su familia. Lo anterior no necesariamente es aplicable a todos los jóvenes, pero quienes se encuentran inmersos en familias con tradición o tendencia al emprendimiento, son más proclives a replicar los patrones que han visto durante su vida, mientras quienes no cuentan con este antecedente pueden presentar mayores dificultades, tanto para decidirse a iniciar como para desarrollar el negocio. Tener conocimiento del contexto socioeconómico del país, el gobierno y las cámaras empresariales es considerada uno de los tres aspectos más importantes al momento de iniciar un negocio. Dato que resulta importante ya que no se puede actuar con base en iniciativas estériles (García-García, 2015:1232).

En cuanto a las actividades que realizan actualmente están: atender su negocio 67\% Del 33\% restante, se distribuyen entre estudios, labores del hogar y ejerciendo la profesión con la que cuentan. De ellos el 28\% incluso ya no tiene que estar de lleno en su negocio, puesto que, los empleados son los encargados de atenderlo, por eso pueden desarrollar otras actividades.

En cuanto estado civil de los jóvenes, la mayoría son solteros $53 \%$ y el resto casados. Podríamos hablar de que los jóvenes del municipio se interesan por cumplir objetivos en el plano personal y profesional antes de contraer responsabilidades de carácter conyugal. Rodríguez (2010) plantea que el alto índice de nacimientos de mujeres adolescentes constituye un obstáculo adicional para que las mujeres jóvenes se inserten en una trayectoria de trabajo. El 15.5\% de los nacimientos 
correspondió a mujeres de entre 15 y 19 años. Que en un futuro tienen que ocuparse en el mercado laboral.

En ocasiones los mismos pobladores de un lugar pueden no identificar las oportunidades, siendo los recién llegados los que aprovechan la situación, por ello se considera importante saber, si los jóvenes beneficiados son originarios del municipio. El 67\% de los cuestionados son originarios del municipio, mientras que los restantes, son procedentes de otras entidades, pero radicados en Colima desde hace tiempo. Esta situación se debe al conocimiento de los programas de apoyo al emprendurismo como lo señala Rodríguez (2010). Lo anterior nos habla de que los mismos pobladores del municipio son quienes están aprovechando los programas, y los que identifican que negocios pudieran ser los adecuados a desarrollar, de acuerdo a las particularidades del lugar.

El 90.5\% de los jóvenes inicia el negocio de manera individual con el apoyo de padres. No tienden a buscar alianzas fuera del núcleo familiar. Distintivo importante en el desarrollo de local como lo plantea Gallicchio (2004).

Por tanto no se identificó alguna asociación o grupo al cual puedan acudir los jóvenes, si bien no por recursos, si en busca del intercambio de ideas y experiencias, las cuales les vayan ayudando a desarrollar su negocio de mejor manera. Desde luego que no todo sale como se planeó desde un escritorio, es necesario tener en cuenta las particularidades del territorio y para saber el cómo, además de contar con una constante evaluación de la política, puesto que si en determinado momento las cosas no salen como se esperaba existe la posibilidad de corregir algunos aspectos que lleven a mejores resultados.

Primeramente no todos los jóvenes se acercan a estos programas de emprendimiento, puesto que consideran que se trata de acciones demasiado arriesgadas o no se sienten con la confianza de poder llevarlas a cabo, para ello es necesario que existan las campañas de solución del proyecto, para que la población objetivo realmente se involucre. El argumento de García-García (2015) se ubica en esta misma idea, ya que la mayoría de los jóvenes no tienen confianza de que exista una cultura empresarial juvenil.

Otro de los problemas más comunes es la falta de evaluación y seguimiento, puesto que una vez entregado el recurso los funcionarios creen haber terminado con su labor. En el caso del municipio de Colima se hace un acompañamiento al emprendedor hasta la apertura de la empresa o negocio. El financiamiento es uno de los principales obstáculos que enfrentan los emprendedores, puesto que en la mayoría de los casos los jóvenes no cuentan con recursos que los puedan avalar para solicitar créditos en instituciones financieras, por ello estas políticas que además de la formulación del proyecto, ofrecen créditos accesibles brindan una mayor posibilidad de éxito para sus jóvenes. Situación presente en la mayoría de los estudios sobre emprendimiento, García-García (2015: 1232) encontró que fue el principal problema en el $71 \%$ de los casos que estudió.

El apoyo al emprendimiento y la generación de empresas por parte de las autoridades es una clara apuesta a la posibilidad de resolver una problemática social que cada día se presenta en mayor proporción.

Existen diversas experiencias internacionales de éxito, a través de las micro y pequeñas empresas se brinda la oportunidad a los jóvenes de permanecer en su lugar de origen evitando la emigración, se llegan a fortalecer los lazos de pertenencia, con lo cual el deseo de superación ya no sólo pertenece a individuos, sino al territorio en su conjunto.

Se desarrollan redes de cooperación y solidaridad que permiten a las micro empresas sean el instrumento mediante el cual, los jóvenes otrora excluidos del ámbito laboral, pueden incluir a otros que se encontraban en situación similar, es una oportunidad de refrendar la responsabilidad, puesto que ahora son elementos activos dentro del territorio.

Se crea una dinámica interna en el territorio, misma que puede llegar a traspasar las fronteras y extender los beneficios a una mayor cantidad de personas a través de la revalorización de sus productos tradicionales, sus atractivos turísticos; se potencializan sus particularidades territoriales, las cuales engloban al conjunto de elementos que dan vida a ese determinado lugar. 
Desde luego que la sola implementación de la política pública no será suficiente, se hace necesaria la activa participación de la sociedad, es necesario llegar al consenso y tener la convicción de trabajar en equipo de lo contrario difícilmente se podrán resolver las problemáticas de la población. No bastaran los apoyos económicos, técnicos y humanos, si estos no están acompañados de elementos sociales y culturales propios del territorio, es decir, se tienen que respetar las particularidades, de lo contrario, las propuestas pudieran resultar contraproducentes, presentando altas probabilidades de fracasar.

Los jóvenes emprendedores no serán simples entes económicos, sino que la dinámica que se genere a partir de su participación debe impactar en la vida social del espacio territorial donde se lleve a cabo. En la tabla 2 se muestran algunos de los resultados de las encuestas aplicadas a los jóvenes beneficiados de los programas de financiamiento (Tabla 2).

Tabla 2. Beneficiarios del Programa de financiamiento para el emprendimiento juvenil en el Municipio de Colima

\begin{tabular}{|c|c|c|c|}
\hline Nombre de la empresa & Giro & Sector & Empleados \\
\hline XOLOTL & Venta de artesanías & Comercial & 3 \\
\hline TEKNOVATIO & $\begin{array}{l}\text { Venta de accesorios para } \\
\text { computadoras }\end{array}$ & Comercial & 2 \\
\hline ANGIES WEB BOUTIQUE & Venta ropa & Comercial & 4 \\
\hline MOTIVOS & $\begin{array}{l}\text { Venta de accesorios, perfumes, } \\
\text { bolsos }\end{array}$ & Comercial & 4 \\
\hline MARISCOS “CHIRRIS” & Restaurant de Marisco & Servicios & 7 \\
\hline COMIENDO EN LINEA & Venta de publicidad Restaurantes & Servicios & 1 \\
\hline $\begin{array}{l}\text { MEDICINA ESTETICA Y } \\
\text { ANTIENVEJECIMIENTO }\end{array}$ & Consultorio Médico & Servicios & 3 \\
\hline ESTANCIA INFANTIL CIPCI & $\begin{array}{l}\text { Servicios de cuidado y atención } \\
\text { infantil }\end{array}$ & Servicios & 7 \\
\hline REVISTA BIO & Venta de espacios publicidad & Servicios & 2 \\
\hline $\begin{array}{l}\text { PUBLICIDAD IMPRESA DE } \\
\text { COLIMA }\end{array}$ & Venta de espacios publicidad & Servicios & 2 \\
\hline $\begin{array}{l}\text { SERVICIOS } \\
\text { ODONTOLOGICOS } \\
\text { ESPECIALIZADOS }\end{array}$ & Consultorio Odontológico & Servicios & 5 \\
\hline $\begin{array}{l}\text { LAVANDERIA Y } \\
\text { PLANCHADURIA LA } \\
\text { "BURBUJA" }\end{array}$ & Planchado Ropa & Servicios & 1 \\
\hline SALON DE BELLEZA & Servicio y cuidado del cabello & Servicios & 2 \\
\hline CIBER TSUBASA & Renta de equipo de cómputo & Servicios & 3 \\
\hline $\begin{array}{l}\text { ESTETICA Y FACIAL } \\
\text { CORPORAL }\end{array}$ & Centro de belleza y control de peso & Servicios & 2 \\
\hline SMOTHIE BAR & $\begin{array}{l}\text { Venta de comidas y bebidas } \\
\text { saludables }\end{array}$ & Servicios & 2 \\
\hline NUTRIFRUITS & Deshidratado y empaque de frutas & Industria & 6 \\
\hline HERRERIA VELASCO & Elaboración de Herrería & Industria & 3 \\
\hline VERMIWORM & Lombricultura & Industria & 3 \\
\hline EL COLIMOTE & Elaboración de bebidas regionales & Industria & 3 \\
\hline $\begin{array}{l}\text { BEBIDAS SALUDABLE } \\
\text { KARAMBA VITAL }\end{array}$ & Producción de bebidas saludable & Industria & 3 \\
\hline
\end{tabular}

Fuente: Elaboración propia con base en las encuestas aplicadas a los jóvenes emprendedores en el municipio de Colima, abril, 2014. 
Las empresas de los jóvenes del municipio de Colima son encabezadas por el sector servicios y comercial. Es un reflejo de lo que se vive en el municipio, el sector terciario predomina y marca la dinámica económica del lugar.

Se aprecia como la mayoría de estas empresas logran generar más de un empleo, incluso se presentan casos donde llegan a generan cinco o más puestos laborales, contribuyendo así a la incorporación de la mano de obra.

Sin embargo, los sectores productivo e industrial se encuentran rezagados, la producción es limitada, donde si bien es cierto, se procura el aprovechamiento de los insumos del territorio, no existen aún empresas de jóvenes que logren generar un producto con alto valor agregado. Dentro de los retos encontrados por el Global Entrepreneurship Monitor (GEM) figura la falta de transferencia de investigación y desarrollo entre los nuevos emprendimientos. Lo cual genera la nula capacidad para adquirir nuevas tecnologías para dar valor agregado a los productos.

Es de destacar primeramente que los medios de difusión del programa son variados, incluso para la convocatoria de este año 2014 se ha recurrido a las redes sociales, en especial a Facebook, se considera que los jóvenes tienen mayor acceso e interacción a través de estas herramientas. En un estudio de García-García (2015) siete de cada diez encuestados en su estudio indicó que no conocían ninguna política o programa gubernamental dirigido al emprendimiento juvenil.

Ante la pregunta ¿Cómo se enteró del programa de apoyo al emprendimiento juvenil? Prevalecen los medios de comunicación e información por conocidos con un 33\% y 38\% respectivamente. Ello nos habla de la difusión que hay del programa de boca a boca, entre los mismos jóvenes se recomienda acudir a la SEJUV en busca de apoyos García-García, 2015:1232-1233 existe un desconocimiento por parte de los jóvenes en torno a las políticas públicas de impulso al emprendimiento empresarial, así como una confusión de lo que se oferta. Los jóvenes mencionaron recordar las campañas impulsadas en la televisión en un 14\%).

En cuanto a la percepción que tienen del programa 57\% de los jóvenes beneficiados califican de bueno el programa, mientras que el 33\% de muy bueno, mencionando que la disposición de los funcionarios de la SEJUV, fue adecuada, sin embargo, mientras en algunos casos el acompañamiento y el seguimiento ha sido constante, en otros ha sido inexistente.

Por ejemplo, el 19\% de los jóvenes comenta que únicamente recibieron apoyo de la SEJUV de carácter financiero, mientras que cerca del $81 \%$ manifiesta que tuvo acceso a servicios de asesoría y acompañamiento, incluso ha recibido visitas de personal de la SEJUV para verificar el estado en el que se encuentra el negocio.

De manera general comentan que desde el acercamiento con la institución fue posible llevar a cabo un proceso conjunto, se desprendió que las asesorías para realizar el trámite fuera satisfactorio. El personal de la SEJUV, les hizo saber de manera oportuna los requisitos y documentos necesarios. Mención especial requiere la elaboración del plan o propuesta de negocio, muchos de los jóvenes manifiestan que no tenían noción de ello, sin embargo, en la SEJUV, encontraron apoyo para elaborarlo de acuerdo a los requerimientos de la convocatoria y poder entregarlo en tiempo y forma. Los jóvenes enfatizan la necesidad de adquirir habilidades clave para el manejo de su nuevo negocio como son: saber tomar decisiones, tener claros los objetivos, capacidad para negociar, ser innovador y creativo, así como contar con la capacidad para detectar oportunidades del negocio (Rodríguez, 2010).

Los trámites para la solicitud del apoyo financiero no requieren de certificación o capacitación por parte de los jóvenes interesados, aunque algunos llevaron el taller "Yo emprendo" su omisión no es motivo de exclusión de la convocatoria.

Aunque el programa tiene como condicionante la aportación por parte del joven del 10\% del capital del proyecto, ya sea en especie o en recursos monetarios, los jóvenes cuestionados no mencionaron esto como una limitante u obstáculo para poder participar, constancia de ello es la aprobación que brindan al programa lo consideran un apoyo importante. Aunque es el país que menos gasta en políticas activas de empleo. A pesar de que el desempleo juvenil está por debajo 
del promedio de la Organización para la Cooperación y el Desarrollo Económicos (OECD) y presenta tendencias a la baja (OECD, 2014).

Otra de las ventajas del programa y que fue identificada como tal por al menos el $19 \%$ de los jóvenes es la prontitud de la entrega del recurso, la convocatoria es de carácter anual, se publica entre los meses de septiembre y octubre. Una vez que se reciben los proyectos y sesiona el comité evaluador, los recursos son entregados a los beneficiarios en el mes de diciembre.

Los jóvenes que se han acercado a la SEJUV en busca de una oportunidad expresan que los apoyos brindados aunque no son suficientes para que el negocio se consolide, si lo son para que inicie. La inversión se complementó en algunos casos con ahorros personales, pero también, cerca del $24 \%$ de los beneficiados requirió financiamiento extra al comienzo del negocio, el cual fue proporcionado por sus familiares. Como sucede en los emprendimientos de las micro y pequeñas empresas en México, en su mayoría se financian por medio de los ahorros familiares (Rodríguez, 2010).

Subrayan al respecto que la familia fue un pilar fundamental en la consolidación de su proyecto, manifestando el apoyo desde diversas esferas, tanto financiero, motivacional, e incluso con mano de obra y conocimientos. Característicos del desarrollo local (Várguez, 2010). Sin embargo, a pesar del apoyo reconocido a la familia, destaca que solo dos de los jóvenes cuestionados reconocen contar con socios formales en la apertura de su negocio. Siendo estos, antiguos conocidos, especialmente compañeros de estudios.

El programa de financiamiento no solo se enfoca a la creación de nuevos establecimientos, algunos de los jóvenes que se han acercado a la SEJUV, lo hacen buscando la ampliación de su negocio, el $24 \%$ manifestó que ya contaban con su negocio, -teniendo en promedio un año de aperturacuando se acercaron a solicitar el apoyo. Incluso hubo quienes lo solicitaron para fortalecer algún negocio familiar, el apoyo brindado por la SEJUV, ha sido aprovechado por los jóvenes para hacerlo extensivo al núcleo familiar. Contrario a lo que encuentra Rodríguez (2010) donde dice que el factor de contagio familiar pro emprendimiento es pequeño, menos de $10 \%$ de los jóvenes encuestados en su trabajo declararon pertenecer a familias con emprendimientos económicos.

El beneficio no se busca de manera individual, en estos casos es involucrar a la familia, pero en otros encontramos que existe asociación con los amigos, mediante el tiempo se desarrollan lazos de compromiso con los trabajadores, los cuales forman parte importante del negocio.

En el aspecto concerniente a calidad de vida, los jóvenes no vacilan al reconocer que esta actividad les ha retribuido un beneficio constante y múltiple, puesto que, reconocen en el emprendimiento, una mejora; primeramente, en su condición económica y social, pero también en el aspecto profesional. Conclusiones a las que también llega García-García (2015:1228) que el emprendimiento coadyuva a la generación de empleos; reducción del desempleo e informalidad juvenil y a la generación de procesos de vinculación en las trayectorias educativo-laborales.

En el económico, a pesar de contar con periodos de altibajos, como todo negocio, mencionan que en términos generales su negocio va a la alza, manifestándose en mayor contratación de empleados, ingresos e incluso en apertura de sucursales. Pero mayormente en la creciente experimentación de independencia económica.

El ámbito social, se fortalece al tener que desarrollar habilidades que les permitan consolidarse, donde al reconocerse como sus propios jefes, las responsabilidades que ello implica los motivan a buscar soluciones en coordinación con las oportunidades que brinda el exterior, aunado a la dosis de reconocimiento, no solo por parte de sus allegados, sino de la sociedad en general que van identificando en ellos potenciales y contribuciones para el municipio. Estas estrategias perfilan a la población mediante las actividades que realizan a crear modelos de gestión para optimizar los recursos con los que tienen a la mano y a su vez dan respuestas a las necesidades del mercado (Rojas y Castellanos, 2014, Citado en García-García, 2015:1228-1229).

El ámbito profesional es mencionado como un segmento que también se ve favorecido (ya sea que cuenten con estudios superiores o no) los constantes retos los obligan a recurrir a mayor 
capacitación, a contar con el manejo de herramientas que les permitan mantener en condiciones óptimas el desarrollo del negocio.

La calidad de vida ha mejorado, porque sus potencialidades se han desplegado ante las implicaciones de abrir un negocio, desde realizar un trámite ante el ayuntamiento o hacienda, hasta colocarse como un negocio competente a la altura de las exigencias del mercado local, pasando por la administración interna y las relaciones laborales. El reto es crear una política de Estado para dar el salto del emprendimiento de subsistencia al de alto potencial de crecimiento y al mismo tiempo hacer un emprendimiento en red que permita generar las economías de escala, mediante la innovación y la competencia (Valencia, 2012:123).

Mencionaron que seguirán adelante en esta actividad como un plan de vida, aunado a los beneficios que reciben de ello, el gusto por desarrollarlo y por los aportes a la comunidad. Algunos de los jóvenes se reconocen como agentes de cambio, capaces de contribuir en la mejora del municipio, no solamente por los empleos que generan, sino por las actividades que desencadenan con su actuar, ya sea entre los proveedores, los clientes e incluso como ejemplo de superación. Podría ser una estrategia adecuada para enfrentar los desequilibrios del mercado laboral que actualmente se padece, para ello se requiere trabajar de forma multisectorial como menciona García-García (2015:1234).

Las principales problemáticas a las que se enfrentan son: recursos insuficientes, colocarse en el mercado, encontrar el local apropiado y en contadas ocasiones los trámites burocráticos. En cuanto a los recursos insuficientes; es una constante al comenzar el negocio, y la principal amenaza para el fracaso, sin embargo, en el municipio de Colima se presenta una cifra favorable, debido a que las empresas juveniles mantienen un alto porcentaje de supervivencia, cerca del $66 \%$ muy por arriba de la media nacional que es del $34 \%$.

Hay que tener en cuenta que, aunque reciban capacitación en manejo de finanzas o administración en la SEJUV, sus capacidades son realmente puestas a prueba conforme el negocio se va desenvolviendo, debido a las malas decisiones o la inexperiencia. Resultado de la falta de oportunidades y la incertidumbre laboral como mencionan Mora y De Oliveira (2011:397).

Lo concerniente a la colocación en el mercado ha sido un problema recurrente, en la mayoría de los negocios existe una amplia competencia, sin embargo, el ser joven puede ser una ventaja, la gente comienza a ver en ellos oportunidad de innovación tanto en los productos como en los servicios ofrecidos.

Tratan de ofrecer algo diferente al consumidor, incluso como plataforma de difusión recurren a redes sociales, muy pocos (14\%) cuentan con página de internet propia, la mayoría (el 52\%) cuenta con un perfil en Facebook, por medio del cual dan a conocer sus productos y servicios. El uso de las tecnologías de la información está presente para dar a conocer los emprendimientos como lo manifiesta García-García (2010).

Con frecuencia el encontrar el local apropiado a las necesidades del negocio, así como un lugar estratégico, y sobre todo los precios accesibles de la renta, son problemas que encuentran los jóvenes justo antes de arrancar con su proyecto. El $72 \%$ renta el local. El emprendimiento requiere de fortalecer y crear una imagen y ubicación de los productos y servicios GEM (2013). Aunque existen múltiples ofertas de recintos comerciales, mencionan que al inicio es difícil poder pagar la renta, para poder competir es necesario ubicarse en zonas estratégicas cuyo valor comercial es elevado. No obstante, algunos terminaron colocando su negocio fuera de estas zonas y comentan que ha sido una buena decisión.

Respecto a los trámites, no es una problemática común, son pocos los jóvenes que realmente se han visto aquejados por ello. La mayoría menciona que incluso les han resultado fáciles. Sin embargo, quienes se han visto agobiados, mencionan que incluso tuvieron que retrasar la apertura porque no les otorgaban la licencia. 
Manifiestan que efectivamente los trámites son simples -como lo promociona el gobierno municipal- incluso son los mismos jóvenes quienes lo realizan, no han tenido necesidad de contratar terceras personas que los auxilien.

En las dependencias son atendidos con eficacia, el mismo personal los auxilia si presentan alguna duda o inconformidad, puntualizan en que estos trámites forman parte de las nuevas responsabilidades adquiridas y como tal deben buscar las maneras de llevarlos a cabo adecuadamente.

En lo concerniente a las nuevas disposiciones fiscales, mencionan que los trámites si han resultado más complicados, ha sido necesario recurrir a los servicios de contadores, pero más allá de la dificultad de los trámites, la queja sentida es contra el sistema de facturación.

Sin embargo, aún son los proveedores locales los que se encargan de suministrar a estos emprendedores, el $62 \%$ de los jóvenes los reconocen como sus principales abastecedores, que genera un efecto dominó en la economía del municipio. El consenso apunta hacia conservar y consolidar el negocio. Ya sea mediante sucursales; con el reconocimiento de su marca o producto; o con la ampliación del ya existente. Mencionan que se han percatado de los beneficios de ser emprendedores, sobre todo en función de las experiencias de sus compañeros de generación o sus amigos cercanos, que son empleados.

Por tanto, se auto reconocen como generadores de cambio, con un fuerte compromiso social, que los motiva a seguir adelante y en la medida de lo posible no desistir de la actividad que ahora desempeñan. Aunado a la satisfacción personal, profesional, económica y social, que ello les provee.

Para la mayoría de los jóvenes cuestionados su negocio actual representa la primera experiencia en este ámbito, aunque, la mayoría con un 52\% coincide en que de no haber recibido los apoyos de la Secretaria de la Juventud (SEJUV), habría buscado otros apoyos, esto se contrapone con la respuesta a otra pregunta, el $95 \%$ de los jóvenes no había pedido un crédito, apoyo o financiamiento anteriormente.

No queda claro entonces, porque no habían decidido iniciar con antelación otro tipo de apoyos, si la idea de un negocio, la determinación y el proyecto ya estaban planeados, ¿Por qué no haber iniciado antes con cualquier otro apoyo?, ya sea de la SEFOME, de la Secretaría de Economía, de la propia Universidad de Colima o incluso créditos de la banca comercial.

Dos de los jóvenes ya contaban con su negocio antes de recurrir al apoyo, esto fue posible según lo expresan por dos factores; sus padres son empresarios y siempre les inculcaron el valor de tomar la iniciativa.

Así como la importancia de generar su propia fuente de ingresos sin importar lo que estudiaran, por lo tanto trabajaron un tiempo al egresar de sus respectivas carreras, lograron generar un capital y con el apoyo de su familia pudieron iniciar sin mayor apoyo extra, hasta la fecha en que lo solicitaron a la SEJUV.

En estos dos casos existieron condiciones para que los jóvenes lograran dar el paso inicial, sin tener que recurrir a más instituciones, pero ¿Por qué específicamente a la SEJUV? Principalmente porque el crédito que ofrecen es blando, y las exigencias son mínimas, contrario a las otras instituciones, como la banca comercial. Como señala GEM (2013) uno de los retos para fortalecer el emprendimiento juvenil es la existencia de una evaluación negativa del país en torno al financiamiento bancario para iniciar nuevos negocios.

\section{Consideraciones finales}

Debido a las transformaciones del mercado de trabajo y pérdida de beneficios laborales adquiridos actualmente. Los jóvenes optan por acogerse a políticas públicas de fomento al emprendurismo, mediante ellas logran sus objetivos personales, sin embargo, durante el proceso de la consolidación de su empresa es posible que lleguen a desarrollar relaciones de solidaridad, las cuales tienen beneficios en el territorio donde se desenvuelve. Pueden ofrecer empleo a la población y activan 
la cadena económica con los proveedores y consumidores. El desarrollo local se ve impulsado por la generación de empleo, que aunque es mínimo puede incentivar sinergias de desarrollo.

El emprendimiento se observa como una oportunidad para revertir las condiciones del desempleo y pudiera fortalecer el sistema productivo del país. Emprenden por necesidad el 61.7\%; y vocación 37.3\% (Rodríguez, 2010), los apoyos surgen de diversas instancias como: gobierno, información escolar y de eventos emprendedores que se dan en su localidad.

Estos nuevos emprendedores son conscientes de las responsabilidades que han adquirido al generar empresas, por ello buscan que el negocio persista y se expanda, aunque carecen de canales de cooperación entre los emprendedores. No existe una red de apoyo entre los jóvenes emprendedores, si está presente entre sus empleados, sus proveedores, clientes e incluso con negocios similares. De esta manera han logrado permanecer en el mercado, mediante el acuerdo con sus proveedores y clientes, generando redes de cooperación que permiten beneficios para las partes involucradas. Pero aun es necesario fortalecer y generar redes de mayor alcance a través de la cual los beneficios sean extensibles a una mayor proporción de la población, logrando consolidar los negocios en menor tiempo y con mejores resultados. Pasar del emprendimiento particular, al emprendimiento cooperativo y colectivo, donde se desarrollen lazos de intercambio, que lograrían trasferencia de información y conocimiento, fortaleciendo a las empresas y actores involucrados en ello, el emprendimiento que genera mayores impactos positivos es aquel que se encuentra ligado a fuertes redes de cooperación. Con lo anterior el desarrollo local del municipio se vería potenciado.

Poco a poco el cambio de paradigma se presenta en los jóvenes, lo cual sin embargo, puede tener múltiples causales, desde el joven que se decide a emprender por curiosidad, por necesidad, por consejo, hasta el que lo ve como su última opción de superación al no encontrar un empleo satisfactorio.

Por ello es necesario fortalecer la cultura emprendedora en el municipio, hacer efectivos los lineamientos de la Ley de Promoción e Impulso al Joven Emprendedor para el Estado de Colima, sobre todo en lo referente a la impartición de talleres y materias que abonen al desarrollo de habilidades y valores encaminados al emprendimiento. En la consolidación de la cultura emprendedora, es necesario que se generen vínculos entre las diversas instituciones, específicamente la Universidad de Colima, el Instituto Tecnológico de Colima y la misma SEJUV, acciones coordinadas entre estos entes tendrán mayores impactos en sus jóvenes. A través de grupos multidisciplinarios es posible llevar a cabo no solo el intercambio, sino la generación de conocimiento que permita consolidar negocios de vanguardia, los cuales repercutirían en beneficio del municipio, es necesario aprovechar las herramientas de las que disponen las instituciones para generar paulatinamente esa sinergia. Perfeccionar los programas de entrenamiento y formación de emprendedores, proporcionándolos a un mayor número de beneficiados ayuda a generar experiencia. Es de destacar que, para que el emprendimiento logre mayores beneficios es necesario se tengan en cuenta elementos como la innovación y la productividad. Los cuales se encuentran presentes en un bajo porcentaje de los proyectos desarrollados, debido a que la mayoría se enfoca a empresas del sector servicios y comercio.

La innovación y lo local deben mantener un vínculo permanente, con base a los elementos propios del territorio es como se van logrando los avances que permiten consolidar los negocios. Ésta relación permite que las acciones generadas tengan un mayor arraigo y tiendan a permanecer y perfeccionarse. No obstante es necesario fortalecer el emprendimiento con acciones complementarias para lograr el desarrollo local, entre las que destacarían; mayor involucramiento entre los productores y proveedores locales, generar un ente capaz de aglutinar a los emprendedores, con lo cual su voz tenga peso en las decisiones gubernamentales, pero también en el ánimo social, lograr un empoderamiento eficaz de los jóvenes. Por parte de las autoridades y las instituciones educativas, sería conveniente realizar análisis en los casos donde las empresas no logran sobrevivir, qué condicionantes las obligaron a cerrar y sí estas pueden tener opciones que 
permitan que perduren. Por tanto la hipótesis que se planteó concluye, que se han generado dinámicas que abonan al desarrollo local, mediante políticas y sus programas pero por si solos aun no son capaces de generar un proceso de verdadero desarrollo local, pero sí constituyen una herramienta importante en la consolidación de éste. Se requiere implantar políticas públicas que implementen plenamente el emprendimiento juvenil.

En la investigación se obtuvieron hallazgos importantes, como el hecho de que los jóvenes elijen el giro de su negocio en función de su experiencia previa como empleados o el gusto por cierta actividad, dejando en segundo plano la posible rentabilidad del negocio. En su mayoría son solteros, con alta escolaridad (nivel licenciatura) en el área de administración y finanzas, sus edades oscilan entre los 23 y 26; establecen redes de cooperación en dos niveles: a nivel familiar (familia nuclear) y personal (amigos y conocidos). Se enfocaron al sector comercio y servicios; tiene una tradición familiar de emprendimiento; El financiamiento es uno de los principales obstáculos que enfrentan los emprendedores, los proveedores son quienes apoyan el desarrollo del negocio al igual que la familia. Se reconocen como participes del desarrollo del municipio, están convencidos de que con su proceder y con mayores apoyos es posible generar beneficios para la población.

Fue posible identificar que los programas no están llegando a sectores marginados, con la inexistente participación de jóvenes con baja escolaridad, lo cual requiere especial atención por parte de las autoridades, identificar por qué no acude a estos programas, si es por decisión propia, por falta de interés o por falta de información. Así mismo, es necesario dar cumplimiento a lo estipulado en la Ley de Promoción e Impulso al Joven Emprendedor.

Existe una selectividad del mercado laboral, mediante su percepción no todos los jóvenes se acercan a estos programas de emprendimiento, puesto que consideran que se trata de acciones demasiado arriesgadas o no se sienten con la confianza de poder llevarlas a cabo, para ello es necesario que existan las campañas de solución del proyecto, para que la población objetivo realmente se involucre.

\section{Bibliografía}

Aguilar, Luis; Mariñez, Freddy; Garza, Vidal. (2009). Política pública y democracia en América Latina del análisis a la implementación. Porrúa, México.

Balaguer, Silvia; Hidalgo, Álvaro; Pérez, Santiago; Pérez, Elena. (2009). Emprendimiento económico y social en España. Guía de recursos para jóvenes emprendedores/as. Instituto de la Juventud, España.

Cardozo, Myriam. (1993). La evaluación de las políticas públicas: problemas, metodologías, aportes y limitaciones. Revista de Administración Pública, Volumen. 84. México. (Pp. 167-197).

Encuesta Nacional de Ocupación y Empleo. (2013) ENOE. INEGI Recuperado de https://www.inegi.org.mx/app/ageeml/ Consulta 10/07/2014.

Gallicchio, Enrique. (2004). El desarrollo local en América Latina. Estrategia política basada en la construcción de capital social. Programa de Desarrollo Local Centro Latinoamericano de Economía Humana (CLAEH) Uruguay. Extraído de http://www.bvsde.paho.org/bvsacd/cd52/capital.pdfConsulta 7/10/2017.

García-García, Víctor. (2015).Emprendimiento Empresarial Juvenil: Una evaluación con jóvenes estudiantes de universidad. Revista Latinoamericana de Ciencias Sociales, Niñez y Juventud, Volumen 13, No., 2. Colombia. (1221-1236).

Global Entreprenurship Monitor (GEM) (2013) Monitor Global de la Actividad emprendedora. México. Recuperado de http://www.gemconsortium.org/report Consulta 11/11/2019.

González, Luis. (2007). Notas sobre Proyectos de Emprendimiento Juvenil en América Latina y Caribe. Proyecto Prejal, Organización Internacional del Trabajo. Extraído de http://www.zerbikas.es/wp-content/uploads/2015/09/1601GONpro.pdf Consulta 28/09/2017. 
Mora, Minor; De Oliveira, Orlandina (2011) Jóvenes mexicanos en medio de la crisis económica: los problemas de la integración laboral. Socieda e Estado. Volumen 26. No., 2. Brasilia. Recuperado de http://dx.doi.org/10.1590/S0102-69922011000200017 Consulta 05/04/2017

OCDE. (2014). Panorama del empleo. Recuperado de http://www.oecd.org/centrodemexico/ estadísticas/. Consulta 30/03/2019.

PEDC. (2015). Plan Estatal de Desarrollo del Estado de Colima 2009-2015. Colima, México.

PMDC (2015). Plan Municipal de Desarrollo del Estado de Colima 2009-2015.Colima, México.

Rodríguez, Laura. (2010). Políticas Públicas para promover el empleo juvenil y el emprendurismo de los jóvenes en México. Recuperado de https://educiac.org.mx/pdf/Biblioteca/Juventud y Empleo/005emp juvenil y empre ndedurismo mexico.pdf Consulta 11/11/2019.

Secretaria de la Juventud (SEJUV), (2013) Informe de actividades. Extraído de http://www.colima-estado.gob.mx/transparencia/archivos/Informe-de-ActividadesSecretaria-Juventud-2013.pdf Consulta 22/03/2018.

Valencia, Germán. (2012). Autoempleo y emprendimiento. Una hipótesis de trabajo para explicar una de las estrategias adoptadas por los gobiernos para hacer frente al progreso del mercado. Semestre Económico. Volumen 15, No., 32. Colombia. (Pp. 103-127).

Várguez, Luis. (2010). El desarrollo local en México: aportes teóricos y empíricos para el debate. [Resumen]. Estudios Sociológicos, volumen. 28, No., 82, México. (Pp. 255-260).

Vázquez, Antonio. (2008). Desarrollo endógeno. Teorías y políticas de desarrollo territorial. Revista Investigaciones Regionales, núm. 11, Universidad Autónoma de Madrid. Extraído de https://www.redalyc.org/articulo.oa?id=28901109 Consulta 21/02/2018. 